\title{
Lifestyle modification for the primary prevention of type 2 diabetes mellitus in the Canadian Aboriginal population
}

\author{
Gabriela Meglei (Meds 2016), Keegan Guidolin (Meds 2017) \\ Faculty Reviewer: Dr Sylvia Orsini, MD, CCFP (Department of Family Medicine)
}

\begin{abstract}
Canada's Aboriginal populations have significantly higher rates of type 2 diabetes compared to non-Aboriginal Canadians. In First Nations populations living on reserve, the rates are more than double. Large randomized controlled trials (RCTs) have shown that intensive lifestyle modification in individuals with impaired glucose tolerance can decrease the overall incidence of diabetes by up to $22 \%$.

Implementing lifestyle interventions into clinical practice remains a significant challenge because of both limited resources and uncertainly about optimal program design. Most studies have focused on translation into the primary care setting, and have shown moderate benefits. However, there have been no trials examining the feasibility and effectiveness of RCT-based lifestyle modification in Canadian Aboriginal communities. Canadian initiatives have so far focused on school-based healthy lifestyle curriculum and community awareness, but have had little success in reducing weight.

Factors such as community remoteness, cultural diversity, poor retention of health care workers, and lack of access to healthy food are significant barriers to implementing lifestyle modification programs in Canadian Aboriginal communities. More importantly, these communities face systemic inequalities that must be addressed in order to achieve meaningful and sustained lifestyle changes.
\end{abstract}

\section{DIABETES IN CANADIAN ABORIGINAL POPULATIONS}

Canada's Aboriginal people include First Nations, Inuit, and Métis and constitute $3.8 \%$ of the population. Although a rare disease in Aboriginal populations prior to the 1940s, the prevalence of type 2 diabetes mellitus (T2DM) in this population has increased by up to $36 \%$ from 2001 to 2006. In $201015.3 \%$ of First Nations living on reserve, and $8.7 \%$ living off reserve reported they had T2DM, compared to $6 \%$ of non-Aboriginal Canadians. Diabetes complications and comorbidities also disproportionately affect Aboriginal people. $^{1}$

Being overweight and obese are chief risk factors for T2DM. In 2010, 74\% percent of First Nations adults living on reserve, and $62 \%$ living off reserve reported they were overweight or obese, compared to $52 \%$ of non-Aboriginal Canadians. ${ }^{1}$ The disparity between Aboriginal people and the rest of Canada is also evident with respect to other T2DM risk factors and health determinants, highlighting the systemic inequalities facing these populations (Table). ${ }^{1-2}$

\section{GOVERNMENT INTERVENTION}

The Canadian government has recognized the diabetes epidemic spreading through First Nations populations. In 1999, Health Canada began the Aboriginal Diabetes Initiative (ADI) as part of the Canadian Diabetes Strategy. ${ }^{3}$ The ADI began Phase 3 in 2012 with $\$ 275$ million of funding over 5 years focusing on initiatives for youth, families, pregnancy and prepregnancy, improved access to healthy food options, and enhanced training for health care providers.

\section{LIFESTYLE MODIFICATION PREVENTS DEVELOPMENT OF T2DM}

Seven large randomized controlled clinical trials (RCTs) have been conducted internationally to examine the effect of lifestyle modification on preventing the development of T2DM in patients with impaired glucose tolerance (IGT). ${ }^{4}$ These interventions were found to reduce the overall incidence of diabetes between 4 to $21.7 \%$ at a follow-up time of 3 to 6 years. ${ }^{4}$

The Diabetes Prevention Program (DPP) in the United States was the largest RCT, and relied on a lifestyle coach and frequent contact to encourage participants to attain a $7 \%$ weight reduction and increase moderate-intensity activity to 150 minutes per week. ${ }^{5}$ The intervention was delivered on an individual basis at a cost of $\$ 1399$ per participant and included 16 sessions and follow-ups. At 3 years, those with intensive lifestyle counseling reduced their relative risk of diabetes by $58 \% .{ }^{6}$ Weight loss was the single most important factor in reducing diabetes incidence. ${ }^{7}$

Unfortunately, several studies reported that the effects of lifestyle interventions decreased in the long term, and the only study that monitored mortality showed no effect on this outcome. ${ }^{4} \mathrm{Al}-$ though lifestyle modification shows promising results, these studies highlight the difficulty of implementing lasting lifestyle changes in high risk populations, even in highly controlled settings.

\section{TRANSLATING PRIMARY PREVENTION STRATEGIES}

The Canadian Diabetes Association Clinical Practice Guidelines (CDA-CPGs) recognize that Aboriginal peoples are at high risk for diabetes and recommend the use of structured lifestyle modification or pharmacological therapy to reduce this risk. ${ }^{8}$ Aboriginal children should be evaluated for modifiable risk factors, prediabetes, and metabolic syndrome. ${ }^{9}$ Screening for diabetes should be started early (age 10 or established puberty) and be more frequent (every 1-2 years) when one or more additional risk factor(s), such as excess weight, abdominal obesity, hypertension, or exposure to diabetes in utero, are present. ${ }^{10}$ 


\section{HEALTH PROMOTION}

Table: Determinants of Health in Aboriginal and Non-Aboriginal Canadians ${ }^{\mathrm{a}}$

HEALTH DETERMINANTS

FIRST NATIONS

(ON-RESERVE)

ABORIGINAL TOTAL NON-

Daily smoking (\%)

46

ABORIGINAL

ABORIGINAL

Smoked during pregnancy (\%)

37

41

22

Dwellings requiring major repairs (\%)

34

19

Living in crowded dwellings (\%)

8

Food insecurity or compromised diet (\%)

Unemployment Rate (\%)

Employment Rate (\%)

Did not graduate high school (\%)

College certificate / university certificate / bachelor's degree (\%)

Incidence of low income in year 2000

Living in families (\%)

Unattached individuals (\%)

Unmet health care needs in last 12 months

Northern Territories (\%)

Canada (\%)

Contact with general practitioner in the last 12 months

Northern Territories (\%)

Canada (\%)

77

Contact with nurse in the last 12 months

Northern Territories (\%)

${ }^{a}$ Adapted from Reading \& Wein, 2009.

${ }^{b}$ First Nations living on reserve and off reserve.

${ }^{\mathrm{c}}$ Inuit.

The implementation of RCT-based interventions into clinical practice or community settings poses many challenges and is in the early stages in Canada. However, studies have shown that intensive lifestyle interventions are cost-effective in patients with IGT, leading to a cost saving of $\$ 84,700$ per quality-adjusted life-year (QALY) for a median cost of $\$ 1,500$ per QALY. ${ }^{11}$ Below we will summarize implementation attempts in different settings and highlight some lessons learned. Finally, we will identify some important factors to consider for implementation in Aboriginal communities.

\section{TRANSLATION TO PRIMARY CARE}

The clinical setting is the most common site for implementing the lifestyle modifications described in RCTs. Adaptation of these interventions into routine clinical practice has required shorter program duration, delivery through group sessions, and intermittent support during the maintenance phase. ${ }^{12}$ Challenges to program implementation include recruiting patients, preventing dropout, determining the optimal mode of intervention delivery, and achieving a sustained lifestyle change. ${ }^{13-14}$ Interestingly, motivational interviewing, a key component of the DPP important in providing resolve to change, is not included in most translational programs.

Several reviews have concluded that implementing lifestyle modifications was feasible and achieved some benefits (eg mean loss at 1 year of $1.8 \mathrm{~kg}^{12}, 4 \%{ }^{13}$ weight loss). ${ }^{12-15}$ Unfortunately, the impact from translational studies was less pronounced compared to that seen in RCTs like the DPP, which achieved a 5 to $7 \%{ }^{16}$ weight loss, and there were small or no improvements in fasting plasma glucose or glucose tolerance. ${ }^{12}$ Diabetes incidence was usually not measured; however, we know from the DPP that a $5 \mathrm{~kg}$ weight loss is estimated to cut the incidence of diabetes in half at 3 years of follow-up. ${ }^{7}$ Therefore, the weight loss achieved in translational studies can also be expected to elicit some reduction in diabetes incidence.

A meta-analysis found the number of sessions attended correlated with increased weight loss and longer programs were likely more effective. Delivery of intervention by lay community staff, use of electronic media, and the absence of a maintenance phase did not have 
a significantly negative impact on weight loss-important findings, given limited resources in rural and Aboriginal communities. ${ }^{13}$

\section{THE CANADIAN PRIMARY CARE EXPERIENCE- "AN OUNCE OF PREVENTION"}

"An Ounce of Prevention" was the first study to implement the DPP into primary care in Canada. ${ }^{17}$ The 4 -week program consisted of a weekly 2-hour session, and was led by a registered nurse or dietician who delivered 90 minutes of education followed by 30 minutes of guided exercise. The cost of the program was $\$ 470$ per participant. Challenges identified included the need for strong endorsement from clinical and administrative leadership to attain optimal space, staffing and recruitment, and the need for ongoing program evaluation.

\section{TRANSLATION TO ALTERNATIVE SETTINGS}

Translation studies have also examined the implementation of lifestyle interventions in community centers (eg YMCA), workplaces, and churches. These settings exhibit greater demographic diversity compared to primary care and may be a better model of how to deliver interventions to Aboriginal communities. Unfortunately, settings with the greatest diversity demonstrated lower, though still statistically significant, weight loss, and less session attendance compared to primary care. ${ }^{15}$

Whereas primary care may utilize existing relationships with health care providers to enhance efficacy and decrease attrition, they are limited by staff and space availability. ${ }^{15}$ These problems are magnified in rural communities because primary care may be unavailable or unable to meet existing health care demands. Community settings use existing structures for program implementation, increase reach in rural communities, and can make use of volunteers.

\section{TRANSLATION TO ABORIGINAL COMMUNITIES}

Few studies have implemented lifestyle interventions into Canadian Aboriginal communities. ${ }^{18-20}$ Challenges to implementation include rural and remote community isolation and unique geography, cultural diversity, low retention of health care professionals, poor continuity of care, lack of appropriate settings for physical activity, unsustained funding, and lack of access to healthy food. ${ }^{21}$

The CDA-CPGs recommend collaborative strategies to engage entire communities and build trusting relationships, incorporation of traditions and local culture, and use of existing community infrastructure when implementing lifestyle programs. ${ }^{9}$ However, some argue that the CDA-CPGs fall short on how these goals should be achieved, and instead recommend that communities be engaged to develop their own unique practice guidelines. ${ }^{21}$ This requires learning about the community and engaging with the chief and council, local health care professionals, and the wider community for support, design, and implementation of programs.

Despite the largely inclusive nature of Canadian interventions, results have been disappointing. One explanation might be the overemphasis on changing interpersonal determinants of health, rather than systemic and environmental factors hindering change in these communities. $^{22}$

In the United States, government funding for the Special Diabetes Program for Indians Diabetes Prevention supported the im- plementation of a 16-session program delivered to 80 tribes in rural, reservation, and urban settings. ${ }^{23}$ Program materials were translated to tribal languages and adapted to local culture. The study reported the average weight loss over 3 annual visits to be $2.5,1.4$, and $1.1 \mathrm{~kg}$, respectively. Since the use of controls was considered unethical, it is uncertain whether the $4 \%$ incidence of diabetes among study participants represents a true decrease in incidence. ${ }^{24}$

\section{THE CANADIAN ABORIGINAL EXPERIENCE}

No RCT-based interventions have been implemented in Canadian Aboriginal communities. Lifestyle programs involving inclusive community consultation have been implemented on the Sandy Lake reserve, ${ }^{25}$ in seven northwestern Ontario First Nations communites, ${ }^{26}$ in the Kahnawake school district, ${ }^{27}$ and in the rural Okanagan region. ${ }^{28}$ These programs have focused on school children and the community at large, and have aimed to increase healthy living skills through a school-based curriculum, promotion of healthy food choices in stores, mass media campaigns, and community events. Unfortunately, despite some studies showing an increase in knowledge, none reported a change in weight or BMI.

Although community-based interventions have a broader reach, the lack of a targeted, intensive approach may have been a factor in the programs' limited success. Participants and staff identified earlier and sustained school intervention, one-on-one communication, increased community awareness, and lower prices for healthy food as ways of improving the program. ${ }^{29}$

\section{CONCLUSIONS}

Despite the largely disappointing outcomes of lifestyle interventions for the prevention of T2DM in Aboriginal communities, a few things are certain. First, Aboriginal communities face many systemic challenges that complicate the already lofty task of achieving sustained individual lifestyle changes through counseling. Second, community ownership and consultation is necessary but not sufficient for programs to deliver measurable risk reductions. Lastly, to achieve a sustained reduction in T2DM incidence, complementary strategies addressing systemic inequalities must be developed, implemented, and evaluated.

\section{REFERENCES}

1. Public Health Agency of Canada. Diabetes in Canada: Facts and figures from a public health perspective [Internet]. Ottawa (ON): Public Health Agency of Canada; 2011 [cited 2013 Jan 22]. 126 p. Available from: http://www.phac-aspc.gc.ca/cd-mc/publications/ diabetes-diabete/facts-figures-faits-chiffres-2011/index-eng.php

2. Reading $\mathrm{C}$, Wien $\mathrm{F}$. Health inequalities and social determinants of Aboriginal Peoples' health [Internet]. Prince George (BC): National Collaborating Centre for Aboriginal Health; 2009 Jan [cited 2013 Jan 22]. 36 p. Available from: http://www.nccah-ccnsa.ca/ en/publications.aspx? sortcode $=2.8 .10$ \&publication $=46$

3. Health Canada. Aboriginal Diabetes Initiative Program Framework 2010-2015 [Internet]. Ottawa (ON); Health Canada; 2011 [cited 2013 Jan 22]. 22 p. Available from: http:// www.hc-sc.gc.ca/fniah-spnia/pubs/diseases-maladies/_diabete/2010-2015-frame-cadre/index-eng.php 
4. Yoon U, Kwok LL, Magkidis A. Efficacy of lifestyle interventions in reducing diabetes incidence in patients with impaired glucose tolerance: a systematic review of randomized controlled trials. Metabolism. 2013 Feb;62(2):303-14.

5. Diabetes Prevention Program (DPP) Research Group. The Diabetes Prevention Program (DPP): description of lifestyle intervention. Diabetes Care. 2002 Dec;25(12):2165-71.

6. Knowler WC, Barrett-Connor E, Fowler SE, Hamman RF, Lachin JM, Walker EA, Nathan DM; Diabetes Prevention Program Research Group. Reduction in the incidence of type 2 diabetes with lifestyle intervention or metformin. N Engl J Med. 2002 Feb;346(6):393-403.

7. Hamman RF, Wing RR, Edelstein SL, Lachin JM, Bray GA, Delahanty L, Hoskin M, Kriska AM, Mayer-Davis EJ, Pi-Sunyer X, Regensteiner J, Venditti B, Wylie-Rosett J. Effect of weight loss with lifestyle intervention on risk of diabetes. Diabetes Care. 2006 Sep;29(9):2102-7.

8. Ransom T, Goldenberg R, Mikalachki A, Prebtani APH, Punthakee Z. Canadian Diabetes Association 2013 Clinical Practice Guidelines for the Prevention and Management of Diabetes in Canada: Reducing the Risk of Developing Diabetes. Can J Diabetes. 2013 Apr;37(suppl 1):S16-9.

9. Harris SB, Bhattacharyya O, Dyck R, Hayward MN, Toth EL. Canadian Diabetes Association 2013 Clinical Practice Guidelines for the Prevention and Management of Diabetes in Canada: Type 2 Diabetes in Aboriginal Peoples. Can J Diabetes. 2013 Apr;37(suppl 1):S119-96.

10. Ekoé JM, Punthakee Z, Ransom T, Prebtani APH, Goldenberg R. Canadian Diabetes Association 2013 Clinical Practice Guidelines for the Prevention and Management of Diabetes in Canada: Screening for Type 1 and Type 2 Diabetes. Can J Diabetes. 2013 Apr;37(suppl 1):S12-5.

11. Li R, Zhang P, Barker LE, Chowdhury FM, Zhang X. Cost-effectiveness of interventions to prevent and control diabetes mellitus: a systematic review. Diabetes Care. 2010 Aug;33(8):1872-94.

12. Cardona-Morrell M, Rychetnik L, Morrell SL, Espinel PT, Bauman A. Reduction of diabetes risk in routine clinical practice: are physical activity and nutrition interventions feasible and are the outcomes from reference trials replicable? A systematic review and meta-analysis. BMC Public Health. 2010 Oct;10:653.

13. Ali MK, Echouffo-Tcheugui J, Williamson DF. How effective were lifestyle interventions in real-world settings that were modeled on the Diabetes Prevention Program? Health Aff (Millwood). 2012 Jan;31(1):67-75.

14. Johnson M, Jones R, Freeman C, Woods HB, Gillett M, Goyder E, Payne N. Can diabetes prevention programmes be translated effectively into real-world settings and still deliver improved outcomes? A synthesis of evidence. Diabet Med. 2013 Jan;30(1):3-15.

15. Whittemore R. A systematic review of the translational research on the Diabetes Prevention Program. Transl Behav Med. 2011 Sep;1(3):480-91.

16. Diabetes Prevention Program Outcomes Study Research Group. Protocol for the Diabetes Prevention Program Outcomes Study [Internet]. Version 3.3. Rockville (MD): George Washington University Biostatistics Center; 2013 Feb [cited 2013 Jan 22]. 93 p.
Available from: http://www.bsc.gwu.edu/dpp./protocol.htmlvdoc

17. Liddy CE, Cullen-Arseneau P, Merizzi S, Blazhko V. "An ounce of prevention": a primary care based prevention program for pre-diabetic population. Can J Diabetes. 2013 Feb;37(1):12-7.

18. McNamara BJ, Sanson-Fisher R, D’Este C, Eades S. Type 2 diabetes in Indigenous populations: quality of intervention research over 20 years. Prev Med. 2011 Jan;52(1):3-9.

19. Teufel-Shone NI, Fitzgerald C, Teufel-Shone L, Gamber M. Systematic review of physical activity interventions implemented with American Indian and Alaska Native populations in the United States and Canada. Am J Health Promot. 2009 Jul-Aug;23(6):S8-32.

20. Edwards K, Patchell B. State of the science: a cultural view of Native Americans and diabetes prevention. J Cult Divers. 2009 Spring;16(1):32-5.

21. Shubair MM, Tobin PK. Type 2 diabetes in the First Nations population: a case example of clinical practice guidelines. Rural Remote Health. 2010 Oct-Dec;10(4):1505.

22. Lévesque L, Guilbault G, Delormier T, Potvin L. Unpacking the black box: a deconstruction of the programming approach and physical activity interventions implemented in the Kahnawake Schools Diabetes Prevention Project. Health Promot Pract. 2005 Jan;6(1):64-71.

23. Jiang L, Manson SM, Beals J, Henderson WG, Huang H, Acton KJ, Roubideaux Y; Special Diabetes Program for Indians Diabetes Prevention Demonstration Project. Translating the Diabetes Prevention Program into American Indian and Alaska Native communities: results from the Special Diabetes Program for Indians Diabetes Prevention demonstration project. Diabetes Care. 2013 Jul;36(7):2027-34.

24. Knowler WC, Ackermann RT. Preventing diabetes in American Indian communities. Diabetes Care. 2013 Jul;36(7):1820-2.

25. Saksvig BI, Gittelsohn J, Harris SB, Hanley AJ, Valente TW, Zinman B. A pilot school-based healthy eating and physical activity intervention improves diet, food knowledge, and self-efficacy for native Canadian children. J Nutr. 2005 Oct;135(10):2392-8.

26. Ho LS, Gittelsohn J, Rimal R, Treuth MS, Sharma S, Rosecrans A, Harris SB. An integrated multi-institutional diabetes prevention program improves knowledge and healthy food acquisition in northwestern Ontario First Nations. Health Educ Behav. 2008 Aug;35(4):561-73.

27. Paradis G, Lévesque L, Macaulay AC, Cargo M, McComber A, Kirby R, Receveur O, Kishchuk N, Potvin L. Impact of a diabetes prevention program on body size, physical activity, and diet among Kanien'keha:ka (Mohawk) children 6 to 11 years old: 8-year results from the Kahnawake Schools Diabetes Prevention Project. Pediatrics. 2005 Feb;115(2):333-9.

28. Daniel M, Green LW, Marion SA, Gamble D, Herbert CP, Hertzman C, Sheps SB. Effectiveness of community-directed diabetes prevention and control in a rural Aboriginal population in British Columbia, Canada. Soc Sci Med. 1999 Mar;48(6):815-32.

29. Rosecrans AM, Gittelsohn J, Ho LS, Harris SB, Naqshbandi M, Sharma S. Process evaluation of a multi-institutional community-based program for diabetes prevention among First Nations. Health Educ Res. 2008 Apr;23(2):272-86. 